\title{
The top quark jet-function at two loops
}

\author{
Ambar Jain \\ Center for Theoretical Physics, Massachusetts Institute of Technology, Cambridge, MA 02139 \\ E-mail:ambarj@mit.edu
}

\section{Ignazio Scimemi ${ }^{* \dagger}$}

Center for Theoretical Physics, Massachusetts Institute of Technology, Cambridge, MA 02139

Departamento de Fìsica Teòrica II, Universidad Complutense de Madrid, 28040 Madrid, Spain

E-mail: scimemi@mit.edu

\section{lain W. Stewart}

Center for Theoretical Physics, Massachusetts Institute of Technology, Cambridge, MA 02139

E-mail:iains@mit.edu

Far above threshold the production process $e^{+} e^{-} \rightarrow t \bar{t}$ can be analyzed using effective field theories. In this talk we consider the invariant mass distribution of top-jets and report about our computation of the two-loop heavy quark jet-function. This is a key part of a next-to-next-to-leading order analysis, and already allows for a resummation of all large logs which effect the shape of the top-invariant mass distribution at next-to-next-to-leading log order. A top-mass scheme is defined which is suitable for measurements involving jets, and whose anomalous dimension is determined by the cusp-anomalous dimension to all orders in perturbation theory.

8th International Symposium on Radiative Corrections (RADCOR)

October 1-5 2007

Florence, Italy

\footnotetext{
* Speaker.

${ }^{\dagger}$ MIT-CTP Preprint number 3931
} 


\section{Introduction}

The era of LHC is now starting and with it some new questions arise about the possibility of measuring the fundamental parameters of the Standard Model (SM) with high precision. The issue is particularly relevant for the top quark mass. The latest Tevatron analysis give $m_{t}=170.9 \pm$ 1.8 [3], a measurement at $1 \%$ level whose precision affects heavily the actual constraints on the Higgs mass as well as many new physics scenarios.

The questions we want to address are the following: i) what observable is both sensitive to the mass of the top quark and under good theoretical control? ii) What is the theoretical framework where we can systematically describe this observable with high precision? iii) How precise is our theoretical control over perturbative shifts to the peak of the invariant mass distribution? iv) And finally, using the invariant mass for a top-mass measurement, what is the most appropriate (stable) mass definition to use, and how well can one related this mass to other existing top-mass definitions? The former 2 points were extensively discussed in the talk of A. Hoang [4] at this meeting. The latter 2 are the focus of this proceedings, for which a complete discussion is given in ref. [6].

We concentrate on $e^{+} e^{-} \rightarrow t \bar{t}$ far from threshold, where the center-of-mass energy $Q^{2} \gg m_{t}^{2}$. The theoretical framework is based on effective field theories like HQET and SCET [5], and has been outlined in ref. [1,2]. Here the top quark decay products form well separated collinear jets together with soft-radiation among the jets. A thrust axis can be defined and this axis define a plane which divides the space in two hemispheres (that we call "a" and "b" hemispheres). A suitable observable is the event-shape cross-section $d^{2} \sigma / d M_{t}^{2} d M_{\bar{t}}^{2}$. Here $M_{t}^{2}=\left(\sum_{i \in a} p_{i}^{\mu}\right)^{2}$ and $M_{\bar{t}}^{2}=$ $\left(\sum_{i \in b} p_{i}^{\mu}\right)^{2}$ are hemisphere invariant masses. The different physics components of $d^{2} \sigma / d M_{t}^{2} d M_{\bar{t}}^{2}$ can be separated by a factorization theorem derived in Ref. [1]

$$
\begin{gathered}
\frac{d \sigma}{d M_{t}^{2} d M_{\bar{t}}^{2}}=\sigma_{0} H_{Q}\left(Q, \mu_{m}\right) H_{m}\left(m, \frac{Q}{m}, \mu_{m}, \mu\right) \int d \ell^{+} d \ell^{-} B_{+}\left(\hat{s}_{t}-\frac{Q \ell^{+}}{m}, \Gamma_{t}, \mu\right) B_{-}\left(\hat{s}_{\bar{t}}-\frac{Q \ell^{-}}{m}, \Gamma_{t}, \mu\right) \\
\times S\left(\ell^{+}, \ell^{-}, \mu\right)+\mathscr{O}\left(\frac{m \alpha_{s}(m)}{Q}, \frac{m^{2}}{Q^{2}}, \frac{\Gamma_{t}}{m}, \frac{s_{t}}{m^{2}}, \frac{s_{\bar{t}}}{m^{2}}\right) .
\end{gathered}
$$

In Eq. (1.1) $\sigma_{0}$ is the tree level Born cross section, $H_{Q}$ and $H_{m}$ are hard-functions which encode the perturbative corrections at the scales $Q$ and $m$, where from now on we use $m$ for the mass of the top quark. The invariant mass variables $\hat{s}_{t}$ and $\hat{s}_{\bar{t}}$ are defined as $\hat{s}_{t}=\frac{s_{t}}{m}=\frac{M_{t}^{2}-m^{2}}{m}, \hat{s}_{\bar{t}}=\frac{s_{\bar{t}}}{m}=\frac{M_{\bar{t}}^{2}-m^{2}}{m}$, and the most sensitive region for mass measurements is the peak region where $\hat{s}_{t, \bar{t}} \lesssim \Gamma_{t}+Q \Lambda_{\mathrm{QCD}} / m$. Finally, $B_{ \pm}$in Eq. (1.1) are heavy-quark jet functions for the top quark/antiquark, and $S$ is the soft function describing soft radiation between the jets. Our main focus here will be on the functions $B_{ \pm}$, which are defined in the heavy-quark limit $m_{t} \gg \Gamma_{t}$ using HQET [7, 8]. The soft function $S$ is universal to massless and massive jets and a suitable model can be found in Ref. [9], extending earlier work in Ref. [10].

In this talk we take the first step toward next-to-next-to-leading order (NNLO) for the invariant mass spectrum, $d^{2} \sigma / d M_{t}^{2} d M_{\bar{t}}^{2}$, by computing the top quark jet function at two-loop order. We also give results for the resummation of large logs for this jet function at next-to-next-to-leading $\log$ order (NNLL). This translates into a resummation of all the large logs in the cross-section that can modify the shape of the invariant mass distribution [1]. We introduce a definition of the top jetmass scheme that has a well defined mass anomalous dimension at any order in perturbation theory 
(unlike the definitions based on cutoff first moments or on peak locations). In this jet-mass scheme the quark-mass anomalous dimension is completely determined by the cusp anomalous dimension at any order in perturbation theory.

\section{The heavy quark jet function}

The jet-functions $B_{ \pm}$for the top quark/top anti-quark are identical by charge conjugation, so we will only refer to the computation of $B \equiv B_{+} . B$ is given by the imaginary part of a forward scattering matrix element, $B\left(\hat{s}, \delta m, \Gamma_{t}, \mu\right)=\operatorname{Im}\left[\mathscr{B}\left(\hat{s}, \delta m, \Gamma_{t}, \mu\right)\right]$, where $\mathscr{B}$ are vacuum matrix elements of a time-ordered product of fields and Wilson lines

$$
\mathscr{B}\left(2 v_{+} \cdot r, \delta m, \Gamma_{t}, \mu\right)=\frac{-i}{4 \pi N_{c} m} \int d^{4} x e^{i r \cdot x}\left\langle 0\left|T\left\{\bar{h}_{v_{+}}(0) W_{n}(0) W_{n}^{\dagger}(x) h_{v_{+}}(x)\right\}\right| 0\right\rangle .
$$

Here $v_{+}^{\mu}$ is the velocity of the heavy top quark, and we introduce null-vectors $n^{\mu}$ and $\bar{n}^{\mu}$ so that we can decompose momenta as $p^{\mu}=n^{\mu} \bar{n} \cdot p / 2+\bar{n}^{\mu} n \cdot p / 2+p_{\perp}^{\mu}$. The vectors satisfy $v_{+}^{2}=1$ and $n^{2}=\bar{n}^{2}=0$, and the definition of the Wilson lines in Eq. (2.1) is

$$
W_{n}^{\dagger}(x)=\mathrm{P} \exp \left(i g \int_{0}^{\infty} d s \bar{n} \cdot A_{n}(\bar{n} s+x)\right), \quad W_{n}(x)=\overline{\mathrm{P}} \exp \left(-i g \int_{0}^{\infty} d s \bar{n} \cdot A_{n}(\bar{n} s+x)\right) .
$$

These Wilson lines make $\mathscr{B}$ gauge-invariant and encode the residual interactions from the antitop jet. The HQET fields $h_{v_{+}}$have the leading order Lagrangian

$$
\mathscr{L}_{h}=\bar{h}_{v_{+}}\left(i v_{+} \cdot D-\delta m+\frac{i}{2} \Gamma_{t}\right) h_{v_{+}} .
$$

Here $\Gamma_{t}$ is the top quark total width, obtained from matching the top-decay amplitudes in the standard model (or a new physics model) onto HQET at leading order in the electroweak interactions, and at any order in $\alpha_{s}$. The residual mass term $\delta m$ in Eq. (2.3) fixes the definition of the top mass $m$ for the HQET computations [11], where $\delta m=m_{\text {pole }}-m$. From the definitions in Eq. (2.1) and the Lagrangian in Eq. (2.3) one can deduce a series of properties of the jet function, which state that it is easy to reconstruct $B\left(\hat{s}, \delta m, \Gamma_{t}, \mu\right)$ from $B(\hat{s}, 0,0, \mu)$. In particular in $\mathscr{B}$ the $\hat{s}, \delta m$, and $\Gamma_{t}$ dependence formally occurs only in the combination $\left(\hat{s}-2 \delta m+i \Gamma_{t}\right)$. For this reason it is useful to have a notation for computations done with a zero residual mass term in the Lagrangian, and with zero-width. Thus we define

$$
\begin{aligned}
B(\hat{s}, \delta m, \mu) \equiv B(\hat{s}, \delta m, 0, \mu), & \mathscr{B}(\hat{s}, \delta m, \mu) & \equiv \mathscr{B}(\hat{s}, \delta m, 0, \mu), \\
B(\hat{s}, \mu) \equiv B(\hat{s}, 0,0, \mu), & \mathscr{B}(\hat{s}, \mu) & \equiv \mathscr{B}(\hat{s}, 0,0, \mu) .
\end{aligned}
$$

\section{Non-Abelian exponentiation and jet-mass}

It is possible to rewrite Eq. (2.1) as a matrix element of pure Wilson lines,

$$
\mathscr{B}(2 v \cdot r, \mu)=\frac{i}{2 \pi N_{c} m} \int d x^{0} e^{i v \cdot r x^{0}} \theta\left(x^{0}\right)\left\langle 0\left|\operatorname{tr} T W_{v}^{\dagger}(0) W_{n}(0) W_{n}^{\dagger}(x) W_{v}(x)\right| 0\right\rangle,
$$


where $2 v \cdot r=\hat{s}$, we use the shorthand $x^{0}=v \cdot x$, and the trace tr is over color indices. The definition of $W_{v}$ and $W_{v}^{\dagger}$ is as in Eq. (2.2) with $n \rightarrow v$. Following the steps outlined in the original paper, [6], also the Fourier transform of the jet function comes out as a product of Wilson lines,

$$
B(\hat{s}, \mu)=\frac{1}{2 \pi} \int d y e^{i \hat{s} y} \tilde{B}(y, \mu), \quad \tilde{B}(y, \mu)=\frac{1}{m N_{c}}\left\langle 0\left|\operatorname{tr}\left[\bar{T} W_{n}^{\dagger}(2 y) W_{v}(2 y)\right]\left[T W_{v}^{\dagger}(0) W_{n}(0)\right]\right| 0\right\rangle .
$$

where $y=y-i 0$ to ensure convergence as $\hat{s} \rightarrow \infty$. Due to the non-abelian exponentiation theorem $[12,13], \tilde{B}(y, \mu)$ exponentiates. Thus we can write the result of our two-loop computation as [6]:

$$
\begin{array}{r}
m \tilde{B}(y, \mu)=\exp \left\{\frac{C_{F} \alpha_{s}(\mu)}{\pi}\left(\tilde{L}^{2}+\tilde{L}+\frac{\pi^{2}}{24}+1\right)+\frac{\alpha_{s}^{2}(\mu) C_{F} \beta_{0}}{\pi^{2}}\left[\frac{1}{6} \tilde{L}^{3}+\frac{2}{3} \tilde{L}^{2}+\frac{47}{36} \tilde{L}-\frac{\zeta(3)}{48}+\frac{5 \pi^{2}}{576}+\frac{281}{216}\right]\right. \\
\left.+\frac{\alpha_{s}^{2}(\mu) C_{F} C_{A}}{\pi^{2}}\left[\left(\frac{1}{3}-\frac{\pi^{2}}{12}\right) \tilde{L}^{2}+\left(\frac{5}{18}-\frac{\pi^{2}}{12}-\frac{5 \zeta_{3}}{4}\right) \tilde{L}-\frac{5 \zeta_{3}}{8}-\frac{17 \pi^{4}}{2880}+\frac{7 \pi^{2}}{144}-\frac{11}{54}\right]\right\},
\end{array}
$$

where $\tilde{L} \equiv \ln \left(i e^{\gamma_{E}} y \mu\right)$. The non-abelian exponentiation theorem guarantees that corrections to this result are $\mathscr{O}\left(\alpha_{s}^{3}\right)$ in the exponent, and that these corrections vanish if we take the abelian limit $C_{A} \rightarrow 0$ and $n_{f} \rightarrow 0$. Since the exponent of the abelian result is one-loop exact, we can use it to test the perturbative behavior of different definitions of the top-mass at any desired order in pertubation theory. Choosing an appropriate top-mass definition corresponds to choosing an appropriate $\delta m$. In ref.[6] we explored several possibilities and came to the following definition, which we refer to as the jet-mass scheme

$$
\delta m_{J}=\left.\frac{-i}{2 \tilde{B}(y, \mu)} \frac{d}{d y} \tilde{B}(y, \mu)\right|_{y=-i e^{-\gamma_{E} / R}}=\left.e^{\gamma_{E}} \frac{R}{2} \frac{d}{d \ln (i y)} \ln \tilde{B}(y, \mu)\right|_{i y e^{\gamma_{E}=1 / R}} .
$$

The scheme depends on a parameter $R$, and we must take $R \sim \Gamma_{t}$ in order to satisfy the power counting criteria. Different choices for $R$ specify different schemes, and are analogous to the difference between the MS and $\overline{M S}$ mass-schemes. The scheme in Eq. (3.4) is free from leading renormalon ambiguities [14]. Let us now check that the mass so defined has good transitivity properties. Transitivity is a well-known feature of the $\overline{\mathrm{MS}}$ mass, and implies that we will obtain the same result if we evolve directly from $\mu_{0} \rightarrow \mu_{2}$, or if we first evolve from $\mu_{0} \rightarrow \mu_{1}$ and then from $\mu_{1} \rightarrow \mu_{2}$. Transitivity is guaranteed by any mass-scheme with a consistent anomalous dimension and renormalization group equation. Since in HQET the scale independent $m^{\text {pole }}=m(\mu)+\delta m(\mu)$, the general form for the RGE equation for the mass is

$$
\mu \frac{d}{d \mu} m(\mu)=\gamma_{m}\left[R, m(\mu), \alpha_{s}(\mu)\right], \quad \gamma_{m}=-\mu \frac{d}{d \mu} \delta m(\mu)
$$

where $R$ is a mass dimension- 1 scheme parameter. To all orders in perturbation theory, using Eq. (3.4), the jet-mass anomalous dimension is [6]

$$
\gamma_{m}^{J}=-\frac{d \delta m(\mu)}{d \ln \mu}=-\left.e^{\gamma_{E}} \frac{R}{2} \frac{d}{d \ln \mu} \frac{d}{d \ln (i y)} \ln \tilde{B}(y, \mu)\right|_{i y e^{\gamma_{E}=1 / R}}=-e^{\gamma_{E}} R \Gamma^{\mathrm{c}}\left[\alpha_{s}(\mu)\right]
$$




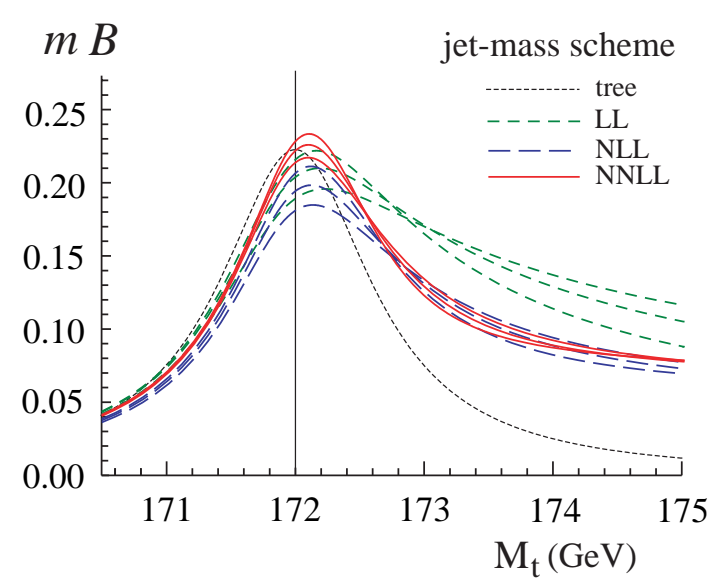

Figure 1: The jet function, $m B\left(\hat{s}, \delta m_{J}, \Gamma_{t}, \mu_{\Lambda}, \mu_{\Gamma}\right)$ versus $M_{t}$, where $\hat{s}=\left(M_{t}^{2}-m^{2}\right) / m$ and $\Gamma_{t}=1.43 \mathrm{GeV}$, and $R=0.8 \mathrm{GeV}$. The black dotted curve is the tree-level Breit-Wigner, the green short-dashed curves are LL results, blue long-dashed curves are NLL, and the solid red curves are at NNLL order. For each order we show three curves with $\mu_{\Gamma}=3.3,5.0,7.5 \mathrm{GeV}$ respectively. Other parameter choices are discussed in [6].

Thus, to all orders in perturbation theory the jet-mass scheme, has a consistent anomalous dimension as in Eq. (3.4), and yields a transitive running mass, $m_{J}(\mu)$. The final anomalous dimension equation for the jet-mass is fully determined by the cusp-anomalous dimension $\Gamma^{\mathrm{c}}$, which is known to three-loop order [15]. Note that the form of the anomalous dimension in $\mu d / d \mu\left[m_{J}(\mu) / R\right]$ has the same structure as that in $\mu d / d \mu[\ln \bar{m}(\mu)]$, where $\bar{m}(\mu)$ is the $\overline{\mathrm{MS}}$ mass.

\section{Results for the NNLL Jet Function}

In this section we discuss the final result for the heavy quark jet function $B\left(\hat{s}, \delta m, \Gamma_{t}, \mu\right)$, with NNLO perturbative corrections and a NNLL resummation of large logs. We have studied the numerical effect of these two-loop corrections as well as of the log-resummation, including the perturbative convergence and $\mu$-dependence of $B$ as a function of $\hat{s}$. At tree-level $B(\hat{s}, \delta m, \mu)=\delta(\hat{s})$ and $B\left(\hat{s}, \delta m, \Gamma_{t}, \mu\right)$ is simply a Breit-Wigner centered at $\hat{s}=0$ with a width $\Gamma_{t}$. Beyond tree-level the jet function becomes dependent on $\mu$ and on the choice of mass-scheme through $\delta m$. For the cross-section $d^{2} \sigma / d M_{t}^{2} d M_{\bar{t}}^{2}$ in Eq. (1.1) it has been proved that at any order in perturbation theory, the only large logs that effect the shape of the invariant mass distribution are those due to the resummation in the heavy-quark jet function [2]. Furthermore these large logs only exist between scales $\mu_{\Gamma} \sim \Gamma \equiv \Gamma_{t}+Q \Lambda_{\mathrm{QCD}} / m$ and $\mu_{\Lambda} \gtrsim \Lambda_{\mathrm{QCD}}+m \Gamma_{t} / Q$. The remaining large logs only modify the cross-section normalization. The expression which sums all logs between the scales $\mu_{Q} \simeq Q \gg \mu_{m} \simeq m \gg \mu_{\Gamma} \simeq \Gamma \gg \mu_{\Lambda} \gtrsim \Lambda_{\mathrm{QCD}}$ is

$\frac{d^{2} \sigma}{d M_{t} d M_{\bar{t}}}=4 \sigma_{0} M_{t} M_{\bar{t}} H_{Q}\left(Q, \mu_{Q}\right) U_{H_{Q}}\left(Q, \mu_{Q}, \mu_{m}\right) H_{m}\left(m_{J}, \mu_{m}\right) U_{H_{m}}\left(Q / m_{J}, \mu_{m}, \mu_{\Lambda}\right)$

$\times \int_{-\infty}^{+\infty} d \ell^{+} d \ell^{-} B_{+}\left(\hat{s}_{t}-\frac{Q \ell^{+}}{m_{J}}, \delta m_{J}, \Gamma_{t}, \mu_{\Lambda}, \mu_{\Gamma}\right) B_{-}\left(\hat{s}_{\bar{t}}-\frac{Q \ell^{-}}{m_{J}}, \delta m_{J}, \Gamma_{t}, \mu_{\Lambda}, \mu_{\Gamma}\right) S\left(\ell^{+}, \ell^{-}, \mu_{\Lambda}, \delta, \bar{\Delta}\left(\mu_{\Lambda}\right)\right)$,

where we have defined the resummed jet function as

$$
B\left(\hat{s}, \delta m_{J}, \Gamma_{t}, \mu_{\Lambda}, \mu_{\Gamma}\right) \equiv \int d \hat{s}^{\prime} U_{B}\left(\hat{s}-\hat{s}^{\prime}, \mu_{\Lambda}, \mu_{\Gamma}\right) B\left(\hat{s}^{\prime}, \delta m_{J}, \Gamma_{t}, \mu_{\Gamma}\right) .
$$


Since the scales $\mu_{\Gamma}$ and $\mu_{\Lambda}$ differ by a factor of $Q / m \gg 1$ it is necessary to sum the large logs between these scales. In Eqs. (4.1,4.2) large logs are resummed by the evolution factors $U_{H_{Q}}, U_{H_{m}}$, and $U_{B}$, and of these, the first two only affect the overall normalization. The numerical importance of the resummation of all large logs was demonstrated at NLL order in Ref. [2].

In fig. 1 we the plot LL, NLL, and NNLL results for the jet function. We observe that the jet-mass scheme results exhibit good perturbative convergence with a stable peak location for the jet-function. In the jet-mass scheme the scale dependence in the slope before the peak is $\sim 6 \%$ at NLL and $\sim 2 \%$ at NNLL, while the maximum variation near the peak is $14 \%$ at NLL and $7 \%$ at NNLL, and then in the tail above the peak it is $\sim 12 \%$ at NLL and $\sim 5 \%$ at NNLL. Thus, in the jet-mass scheme the $\mu_{\Gamma}$ dependence is reduced by a factor of two or more. The same level of improvement is also observed for different mass-scheme parameters $R$ than the one shown.

\section{Acknowledgments}

This work was supported in part by the Department of Energy Office of Nuclear Science under the grant DE-FG02-94ER40818. I. Scimemi was also supported in part by a Marie Curie International Fellowship from the European Union, grant number 021379 (BDECMIT), and thanks the Fundació Bosch i Gimpera of the University of Barcelona (Spain) for support. I.W. Stewart was also supported in part by the DOE Outstanding Junior Investigator program and Sloan Foundation.

\section{References}

[1] S. Fleming, A. H. Hoang, S. Mantry and I. W. Stewart, arXiv:hep-ph/0703207.

[2] S. Fleming, A. H. Hoang, S. Mantry and I. W. Stewart, arXiv:0711.2079 [hep-ph].

[3] Tevatron Electroweak Working Group (2007), hep-ex/0703034.

[4] Talk of Andre Hoang at this same conference.

[5] C. W. Bauer, S. Fleming and M. E. Luke, Phys. Rev. D 63, 014006 (2001); C. W. Bauer, S. Fleming, D. Pirjol and I. W. Stewart, Phys. Rev. D 63, 114020 (2001); C. W. Bauer and I. W. Stewart, Phys. Lett. B 516, 134 (2001); C. W. Bauer, D. Pirjol and I. W. Stewart, Phys. Rev. D 65, 054022 (2002); C. W. Bauer, S. Fleming, D. Pirjol, I. Z. Rothstein and I. W. Stewart, Phys. Rev. D 66, 014017 (2002); C. W. Bauer, C. Lee, A. V. Manohar and M. B. Wise, Phys. Rev. D 70, 034014 (2004).

[6] A. Jain, I. Scimemi and I. W. Stewart, arXiv:0801.0743 [hep-ph].

[7] A. V. Manohar and M.B. Wise, Camb. Monogr. Part. Phys. Nucl. Phys. Cosmol.10,1 (2000).

[8] M. Neubert, Phys. Rept. 245, 249 (1994), hep-ph/9306320.

[9] A. H. Hoang and I. W. Stewart, Phys. Lett. B 660, 483 (2008) [arXiv:0709.3519 [hep-ph]].

[10] G. P. Korchemsky and S. Tafat, JHEP 10, 010 (2000), hep-ph/0007005

[11] A. F. Falk, M. Neubert, and M. E. Luke, Nucl. Phys. B388,363 (1992),hep-ph/9204229.

[12] J. G. M. Gatheral, Phys. Lett. B133, 90 (1983).

[13] J. Frenkel and J. C. Taylor, Nucl. Phys. B246231 (1984).

[14] S. Fleming, A. Hoang, S. Mantry and I.W. Stewart in preparation

[15] S. Moch, J. A. M. Vermaseren and A. Vogt, Nucl. Phys. B 688, 101 (2004) [arXiv:hep-ph/0403192]. 